\title{
The Design of Automatic Batching Device for Anaerobic Fermentation of Fresh Kitchen Waste in Community
}

\author{
Shijie Fang, Wuyingni Li, Xurong $\mathrm{Li}^{*}$, Haoyuan Jiang, Chenhao Zhang, Yuxuan Wu and \\ Donghe Ma \\ College of Engineering, HuaZhong Agriculture University, Wuhan,Hubei, China 430070
}

\begin{abstract}
In this paper, according to the real situation of the treatment of fresh kitchen waste in large and medium-sized communities, and combined with the study that fresh waste can be converted into environmental protection enzyme by anaerobic fermentation, a community fresh kitchen waste treatment device which can automatically complete feed transportation, proportioning ingredients, self-fermentation and regular reminders is proposed.By using hydraulic device and moving pulley conveying chain, single chip microcomputer control, combined with weighing, flow, infrared, Ph detection and other sensing technology, the community kitchen waste automatic treatment, emission reduction and efficiency.
\end{abstract}

\section{Introduction}

The disposal of fresh kitchen waste is still one of the problems that puzzles the subsequent treatment of garbage classification. And community garbage, a large part of the fruit peel, vegetable leaves and other kitchen waste mainly organic waste. At present, this kind of garbage is usually discarded at will or disposed of according to the way of general domestic waste.

This design is a set of large-scale fresh kitchen waste energy-saving and emission reduction device. The device is mainly composed of three systems: garbage bin transportation and dumping unloading system, fresh kitchen waste crushing and pushing system, proportioning and fermentation monitoring electronic control system.It can complete a large community $100 \mathrm{~kg}$ of fresh kitchen waste automatic fermentation. According to the daily amount of fresh kitchen waste produced by the community, the fresh fermentation materials and the capacity of fermentation equipment can be recycled.The power source required for the whole plant is photovoltaic solar panels. In the case of selfsufficiency of electricity, the production of environmental enzymes under automatic control can be completed only by adding sugar regularly.Energy saving and environmental protection, high degree of automation, high feasibility. It improves the utilization rate of garbage,achieves the purpose of energy saving and emission reduction, and strengthens the

*Corresponding author: lunar@mail.hzau.edu.cn 
consciousness of garbage classification of community residents.It can realize the problem of garbage and is beneficial to the environmental protection of the community.

\section{Waste anaerobic fermentation automatic batching device}

Based on the characteristics of community use and community site, the overall design is shown as Figure1. The device mainly includes three parts: automatic lifting and unloading of garbage bin, crushing and pushing of fresh kitchen waste, proportioning and fermentation monitoring electronic control part. The two faucets are connected with water pipe and molasses pipe respectively,and the flow rate is automatically controlled by solenoid valve switch. In order to reduce the cost, molasses liquid can be used instead of brown sugar in community fermentation, and it is convenient for machine to use in large quantities.

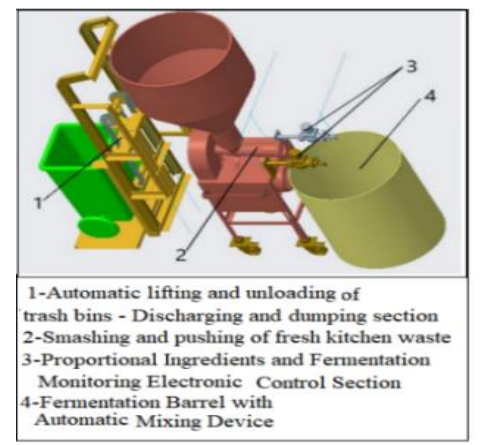

Fig. 1. Schematic diagram of the overall equipment model

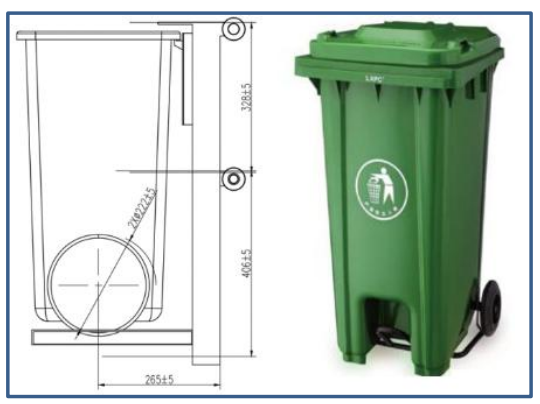

Fig. 2. Community trash bin size

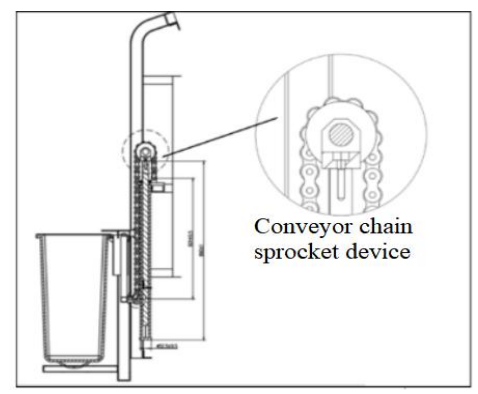

Fig. 3. Automatic unloading device for trash bin

\subsection{Automatic lifting and unloading of dump bins}

According to statistics, the daily production of fresh kitchen waste in most communities is about $100 \mathrm{~kg}$, and a large number of existing community trash bins are shown in Figure 2. If the kitchen waste is concentrated in a trash can, it can account for about $2 / 3$ of the volume of the trash can. In order to realize the automatic dumping of garbage in the bucket, it is necessary to design a reliable large-scale transportation and unloading system. The function of the transport and unloading section is to transport the garbage bin from the ground to the feed port and complete the automatic dumping. This part is designed to avoid traditional man-made handling of trash bins. The traction power adopts a single piston rod hydraulic cylinder to provide stable and high power power output; combines the conveying 
chain with the moving pulley mechanism to shorten the half stroke of the hydraulic cylinder and save the device space; after completing the lifting and dumping action of the trash can, the hydraulic system guides the descent reset. The automatic unloading scheme is shown in Figure 3. Design related parts according to workload and unloading speed.[1]

Garbage can lift trip $1.15 \mathrm{~m}$, Maximum load is set to $3000 \mathrm{~N}$, A weight rises at $0.2 \mathrm{~m} / \mathrm{s}$, The speed V1 of hydraulic rod is $0.1 \mathrm{~m} / \mathrm{s}$. Double gear pump with rated pressure of 7.54 $\mathrm{MPa}$, maximum pressure $10 \mathrm{MPa}$,displacement Speed is $10 \mathrm{ml} / \mathrm{r}$, rotation rate is $600-1500$ $\mathrm{r} / \mathrm{min}$. A steel pipe with a nominal diameter of $12 \mathrm{~mm}$, outer diameter $18 \mathrm{~mm}$, and select connection thread specification for M18 $\times 1.5,2 \mathrm{~mm}$.Using tube wall --2 FDY-P-E100 $\times 20$ FS, hydraulic oil filter.A filtering precision of $20 \mu \mathrm{m}$, it can keep the hydraulic fluid clean, effectively filter out the contaminants in the hydraulic system,prolong the service life of hydraulic pumps, make the hydraulic pump work normally and safely. Y100L-6 type motor is used to drive the hydraulic pump, $1.5 \mathrm{KW}$, rated rated speed $1000 \mathrm{r} / \mathrm{min}$. The selection model of conveying chain is $04 \mathrm{C}$-1, its pitch is $6.35 \mathrm{~mm}$,roller diameter is $3.30 \mathrm{~mm}$, ultimate tensile load is $3.50 \mathrm{KN}$, meeting the design requirements. The conveying chain adopts the form of moving pulley arrangement, which can reduces the lift stroke by half, and can adapt to the harsh environment and has strong reliability. The position arrangement is shown in Figureure 3 attached to the conveying chain sprocket arrangement device.

\subsection{Rack design}

Figure 4 is a load rack for the connection between a common trash can and a delivery rack. Figure 6 is a weighing platform, which is used for carrying and placing garbage bins, and a weighing sensor is arranged at the bottom to weigh the kitchen waste in the bucket statically. The support frame is shown in Figure 7 for the fixing and supporting of the trash bin and the arrangement of the conveying sprocket. The connecting plate, as shown in Figure 8 , is used to limit the dumping position of the trash can to ensure the accurate position of the dumping angle. The guide groove is shown in Figure 5 as the guide rail guide frame for the whole frame.

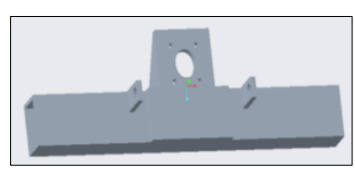

Fig. 4.Bearing frame

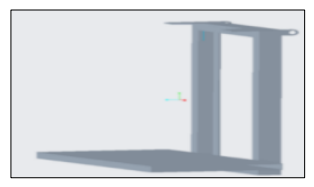

Fig. 6. Weighing table

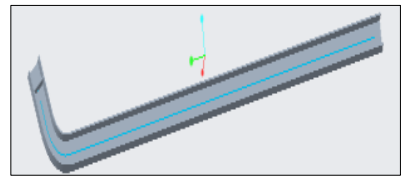

Fig. 5. Guide slot

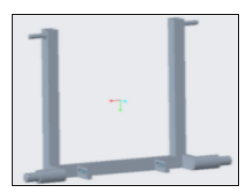

Fig. 7. Supporting frame

\subsection{Feed and shredding design}

The feed hopper is shown in Figure 9 , based on the elastic dynamic model of the vibrating bowl feeder, the bowl type is combined with the cylinder type. There is a special spiral inside, which is used for the movement guidance of the material in the bucket. Reasonable blanking can play the role of anti-blocking. The lower part of the feed hopper is welded 
with the winch installed. The steel frame plane (Figure .10) is used as the supporting platform of this part, and the steel frame is used as the hardware bearing platform of the electronic control room. The function of the dragon is to turn the vertical falling garbage in the feed hopper into horizontal and push the material, and the push process will crush the garbage once. In this part, a variable pitch winch with unequal helical spacing is designed (see Figureure 11). The front (left) end of the pitch is small, the rear (right) end is large, and the root diameter is short and long. The kitchen waste is discharged from the hopper into the large pitch behind the winch, and the winch rotates horizontally to push the extruded material forward. The space volume of the winch and the winch decreases with the pitch, and the material is crushed by shear force. Then pushed to the front-end reamer, reamer rotation can be used for material secondary crushing.

According to the following formula, the material of the dragon is selected as the Cr18Ni9, screw of rust prevention: $\mathrm{D}=\mathrm{K} \sqrt[2.5]{\frac{G}{\varphi \rho C}} \varphi$. The $\mathrm{K}$ represents the comprehensive characteristic coefficient of the material, the $G$ represents the production capacity, the filling coefficient of the material, the $\rho$ represents the packing density of the material, the $\mathrm{C}$ is 1 . The $\mathrm{G}$ is $0.1 \mathrm{t} / \mathrm{h}, \varphi$ is $0.20, \mathrm{~K}$ is 0.071 , and after many tests, $\rho$ is $0.6 \mathrm{t} / \mathrm{m}$., After substituting into calculation. $\mathrm{D}=66.0 \mathrm{~mm}$, Take the $\mathrm{D}$ as $70 \mathrm{~mm}$. The square shaft in the front (right) of the winch is used to install reamers. The surface of the cutter is smooth, the strength is high and the cutting edge is sharp.After reading $<$ Multi-function automatic meat grinder design $>$ [2] In order to have better effiency and more durable usage time, the material with high hardness, not easy to oxidize and very good cutting performance is selected. The quenching strength is HRC55-60. W18Cr4V the cutter as shown in Figure 12 The cutter blade is 4 blade.

\subsection{Other structural design models}

Because the work of squeezing the dragon is continuous, the material is constantly extruded from the front end of the dragon, and the material is crushed twice by the reamer. Because the pressure of the leading edge is increasing continuously, in order to make the extrusion discharge smooth without clogging, the extrusion pressure plate is set at the outlet of the cutter behind the reamer. When the front edge material of the reamer is more than a certain degree, the press plate is pushed out[2], This feed crushing device (Figure .13) has high automation level, simple operation, easy maintenance, large daily handling capacity, low power consumption and suitable for continuous operation.

The arrangement of the winch transmission part is shown in Figure 14. The large belt wheel adopts the form of reinforcement arrangement to meet the stiffness requirements and reduce the weight of the body. Work with the small belt wheel to complete the power transmission of the winch reamer.

\subsection{Control section}

\subsubsection{A/D converter chip}

Select HX711 high-precision 24-bit A/D converter chip. The chip integrates the peripheral circuits needed by other chips of the same type, such as power supply, on-chip clock oscillator and so on. It can reduce the cost of weighing part and improve the performance and reliability of the whole machine. 


\subsubsection{Weighing sensor}

The weighing sensor is arranged at the bottom of the weighing bearing table and needs to complete the static and stable weighing of solid waste. The cantilever parallel beam strain sensor (Figure 15) is selected as the sensing component. The sensor has the advantages of convenient processing, good stability and high precision of weighing.

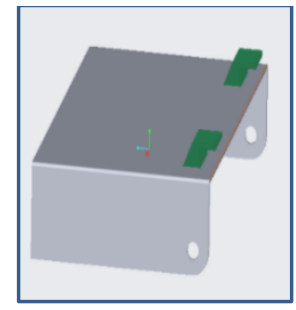

Fig.8. Connecting Plate

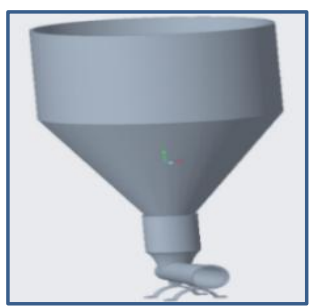

Fig. 9. Feed hopper

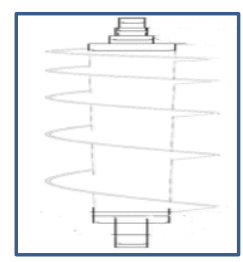

Fig. 11. Variation pitch winch

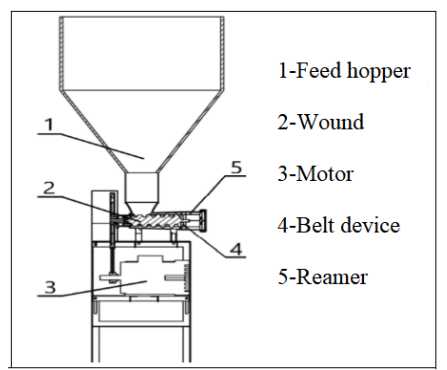

Fig. 13.Design of the feed chipping section

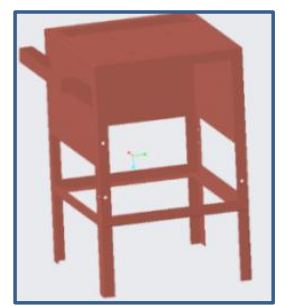

Fig. 10.Steel frame

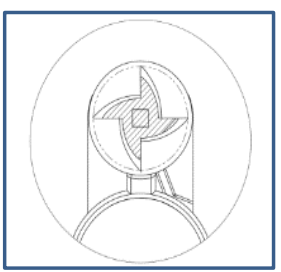

Fig. 12. Reamer

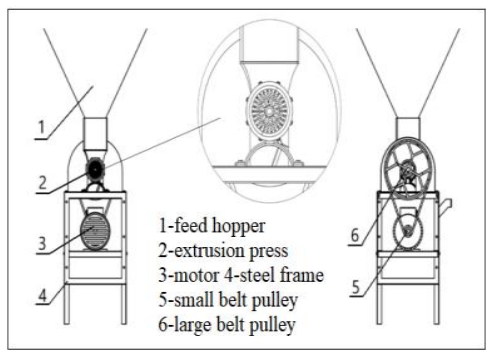

Fig. 14. Design of the winch transmission section

The working principle of cantilever parallel beam is shown in Figure 16. When the elastic beam in the sensor bears the gravity pressure of the trash can, it deforms and makes the strain gauge feel the same deformation, and the sensor converts the deformation into a change of resistance. At this time, the sensor and the amplifier circuit wiring, finally the deformation analog quantity into the digital voltage change, through the voltage change value analysis processing conversion, finally can measure the garbage quality in the current garbage bin, further carries on the proportional calculation, obtains the water, molasses quality ratio, controls the corresponding solenoid valve work.

\subsubsection{Control physical wiring}

In this design, the control experiment design of the proportional feed control part is completed, and the physical object of the control part is made (Figure 17). Many experiments have achieved ideal results. LED lamp indicates solenoid valve state, light indicates solenoid valve on, lamp out indicates solenoid valve off. After the sensor detects 
the weight information of the trash can, the single chip microcomputer receives the signal, calculates the quality corresponding to the effluent and molasses, and opens the solenoid valve to control the flow of water and molasses into the fermentation chamber, respectively. At this time, the LED lamp is always on. When the flow sensor detects that the volume of water and molasses outflow reaches the ratio value, the single chip microcomputer receives the signal again and closes the corresponding solenoid valve, and the LED lamp goes out. In the process of liquid feed (that is, in the process of light on), the automatic mixing device in the fermentation chamber is always in the working state, which ensures that the sugar water is mixed while feeding and mixing, and reduces the difficulty of subsequent stirring and power consumption.

\section{Working principles}

By using flow sensor,ph detection device, exhaust pressure reducing valve, pressure sensor and photovoltaic power generation system, the automatic fermentation process is realized. I want to improve the the traditional method though making improvement on the basis of the present technologies.[3]The main technical problems solved in the design are three problems: automatic lifting feed, automatic proportioning feed and regular cycle fermentation.

\subsection{Automatic feeding principle}

The conveying chain and each supporting chain plate fix the heavy duty garbage can, and through the linear power output of the single piston rod hydraulic cylinder, complete the linear lifting, dumping and falling movement of the community heavy duty garbage can. When the weighing sensor receives a steady weight signal, the single chip microcomputer controls the motor operation connected to the hydraulic pump, and the hydraulic system outputs a stable and large load power, which is guided by the conveying chain to lift and dump the action. This process greatly reduces the participation of manual labor and ensures that the kitchen waste in the heavy trash can is automatically poured into the feed hopper.

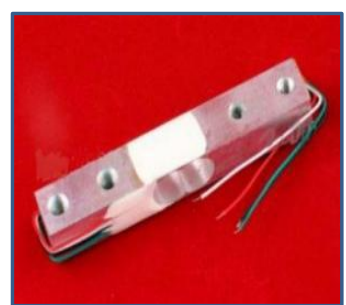

Fig. 15. Two-hole cantilever parallel beam strain sensor

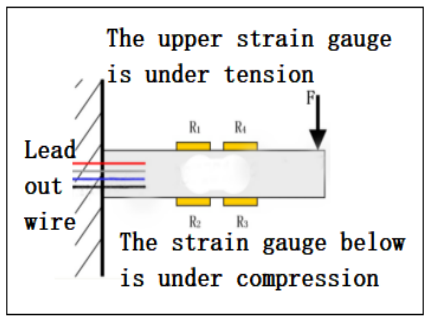

Fig. 16. Working principle of sensor

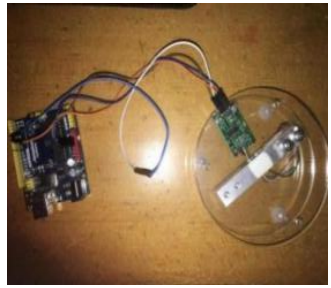

Fig. 17. Physical picture

\subsection{Feed principle of automatic proportioning}

Environmental enzymes are made by mixing molasses, kitchen waste and water in 1:3:10 weight. In the quality calculation of solid fresh garbage, the cantilever parallel beam pressure sensor is used to arrange it on the bottom platform. Before lifting and transporting the material, the net weight of the material can be measured first. Then the quality information is input to the single chip microcomputer. According to the quality of kitchen 
waste, the processor calculates the ratio of effluent and molasses, so as to control the opening and closing of the corresponding solenoid valve. Feed water and molasses to fermentation chamber and complete automatic stirring at the same time.

\subsection{Regular cycle fermentation principle}

After all the feed is finished, the infrared sensor in the fermentation room detects whether the solid material is not fully immersed in the liquid. If there is an submerged material, the automatic mixing device at the bottom of the fermentation room will carry out solid-liquid mixing and stirring. If all solid materials have been immersed in liquid, the fermentation chamber is automatically closed. The fermentation chamber is then transported to the storage center according to the pre-set transmission route. During fermentation, the $\mathrm{Ph}$ detector and automatic mixing device will work regularly to monitor the fermentation situation. When the indoor pressure increases to a predetermined value, the pressure relief valve automatically opens for venting. After 3 months of fermentation, the user is automatically reminded to complete the fermentation. The fermentation chamber can be recycled, and the power supply of the whole plant is provided by photovoltaic solar panels, which is self-sufficient and does not require additional energy consumption.

\section{Description of core components}

\subsection{Trash bin transport and dump system}

Loading and unloading power adopts the stable and large power output provided by the hydraulic cylinder, the lifting is stable and accurate, the load is large, the operation is simple, and it is easy to realize the automatic control of garbage lifting and unloading.Adopt the dynamic pulley chain type lifting, not only the transmission efficiency is high, but also the lifting stroke is halved, which makes the lifting structure compact.Without manual intervention, automatic discharge is realized, and labor is reduced.

\subsection{Fresh kitchen waste shredding and feeding system}

The design of variable pitch winch can complete the lateral push and save the design of transmission platform.By twisting dragon rotation, extrusion and division of solid fresh garbage, complete a material crushing. The reamer is installed at the exit of the dragon to realize the secondary crushing of the material and facilitate the full fermentation.Extrusion plate is arranged the outlet of the winch to make the push material uniform and avoid clogging.

\subsection{Innovation of electronic control system for proportional ingredients and fermentation monitoring}

I habor the idea that if the device is based on the single chip microcomputer, the automatic proportional calculation and control of feed can be realized[4].According to the different amount of fresh kitchen waste each time, the dynamic scientific calculation ratio can be carried out, and the weighing sensor, solenoid valve and flow sensor can work together. The control part is simple and powerful.The fermentation monitoring system is connected with the infrared sensor and $\mathrm{Ph}$ monitor in the fermentation room. It is used to monitor whether any materials in the fermentation room are not submerged by liquid, to prevent the fermentation raw materials from mildew, to monitor the ph value in time, and to prevent the 
fermentation failure. The bottom of the fermentation chamber is equipped with an automatic mixing device, the water and molasses are fully stirred first and then mixed evenly with the solid fresh garbage, which can greatly reduce the output torque and energy consumption of the mixing device. .

Due to the need to maintain a closed environment during fermentation, the fermentation chamber is equipped with a pressure reducing valve, which can automatically adjust the indoor air pressure without the need for artificial open cover decompression.

\subsection{Conception of automated transport storage system for fermentation chambers}

After all the feed work in the 1) fermentation room is completed, it will move to the storage center with the mobile transportation platform, carry on the centralized fermentation, take the liquid, add the visual material collection interactive system, enhance the community residents' experience. Set up different specifications of removable fermentation room, according to the daily amount of garbage matching fermentation room volume. Realize variable flexible fermentation, save space, fermentation chamber sustainable recycling.

\section{Conclusion}

This device starts with the characteristics of classified collection and treatment of fresh kitchen waste in large community, takes the production automation of environmental protection enzyme as the core, and aims to promote the classification and innovative treatment of community kitchen waste. Kitchen waste is taken from the people and turned into treasure for the people. Concentrated treatment is conducive to reducing costs, energy saving and emission reduction, centralized fermentation, help to promote the use of environmental enzymes, improve the environment, reduce pollution. The design has the advantages of circular sustainable development, in line with the purpose of energy saving and low carbon and the development concept of green environmental protection.

\section{References}

[1] Xing Zhigang, Sun Fenghua, Zhao Miao Miao.Application of moving pulley stroke multiplication structure in frame column emulsion drilling rig. Coal Mine Machinery,[J](Haer bin,Hei Long Jiang)2015,36(10):214-215.

[2] Cao Leping, Deng Jiehong. Multi-function automatic meat grinder design.Food and Machinery[J](Changsha:Hunan)2006,22(2):77-80...

[3] Cai Yifei, Tang Min. Study on fermentation process of environmental protection enzyme. Technology Information,[M](Qingdao:Shandong)2017(14):230-230.

[4] Jia Guangzheng, Meng Xiangwei, Zhang Fuchen, Ren Yongliang. Control hydraulic loading system based on PLC proportion[J]. Hydraulic and Dynamic,(Beijing:China) 2010(07):14-16. 